\title{
Fused Video and Ultrasound Images for Minimally Invasive Partial Nephrectomy: A Phantom Study
}

\author{
Carling L. Cheung ${ }^{1,2}$, Chris Wedlake ${ }^{1}$, John Moore ${ }^{1}$, \\ Stephen E. Pautler ${ }^{3}$, and Terry M. Peters ${ }^{1,2}$ \\ ${ }^{1}$ Imaging Research Laboratories, Robarts Research Institute \\ ${ }^{2}$ Biomedical Engineering Graduate Program, The University of Western Ontario \\ ${ }^{3}$ Division of Urology, Department of Surgery and Division of Surgical Oncology, \\ Department of Oncology, The University of Western Ontario, \\ London, Ontario, Canada
}

\begin{abstract}
The shift to minimally invasive abdominal surgery has increased reliance on image guidance during surgical procedures. However, these images are most often presented independently, increasing the cognitive workload for the surgeon and potentially increasing procedure time. When warm ischemia of an organ is involved, time is an important factor to consider. To address these limitations, we present a more intuitive visualization that combines images in a common augmented reality environment. In this paper, we assess surgeon performance under the guidance of the conventional visualization system and our fusion system using a phantom study that mimics the tumour resection of partial nephrectomy. The RMS error between the fused images was $2.43 \mathrm{~mm}$, which is sufficient for our purposes. A faster planning time for the resection was achieved using our fusion visualization system. This result is a positive step towards decreasing risks associated with long procedure times in minimally invasive abdominal interventions.
\end{abstract}

\section{Introduction}

Many abdominal surgery procedures are now performed minimally invasively. In this paper we discuss laparoscopic partial nephrectomy, which is the resection of a renal tumour along with a margin of healthy tissue to ensure cancer control. During this procedure, the surgeon uses a laparoscopic camera to view the organ surface and a laparoscopic ultrasound (US) probe to visualize the tumour and other underlying structures to plan and perform the resection. In the conventional operating room (OR), the video and US images are presented separately and are typically in 2D (Fig. 1a-b). Therefore, the surgeon has to look back and forth between the images and mentally map the US image onto the video image to determine where the tumour is relative to the organ surface. Furthermore, the $2 \mathrm{D}$ nature of the images results in decreased depth perception.

A more intuitive visualization system would be advantageous for guidance during partial nephrectomy, especially in decreasing procedure time and in

T. Jiang et al. (Eds.): MICCAI 2010, Part III, LNCS 6363, pp. 408-415, 2010.
(C) Springer-Verlag Berlin Heidelberg 2010 
achieving a more accurate margin. During partial nephrectomy, the kidney is often subject to warm ischemia [1]. Periods of warm ischemia greater than 30 minutes put the organ at risk of irreparable damage, therefore a short warm ischemic time is crucial. A $1 \mathrm{~cm}$ margin of normal renal parenchyma is accepted as the standard to prevent the recurrence of cancer. More recently, it has been suggested that a smaller margin of $5 \mathrm{~mm}$ or even less is acceptable for cancer control [2]. A smaller margin allows for more healthy tissue to be retained, increasing the nephron sparing and the likelihood of a positive postoperative prognosis. To attain a smaller margin without violating the tumour boundaries, it is crucial to know exactly where the tumour is located and how far it extends into the organ.

We developed and validated a visualization platform (Fig. 1 $\mathrm{k}$ ) that fuses video and US in a common 3D environment. This work is presented as a system that is unique from other work 34 through the use of magnetic tracking and a flexible-tip US probe, combined with stereoscopic video. The goal of this paper is to evaluate the efficacy of fused video and US by studying surgeon performance under the guidance of the conventional setup (video and US separate) and the fusion setup, presented both monoscopically and stereoscopically. This goal is achieved with a novel phantom study. Targeting has been shown to be faster with a stereoscopic visualization [5], and more accurate using multimodality fusion [6]. These studies provide the basis for exploring the use of a stereoscopic fusion environment for more complex tasks such as tumour resection.
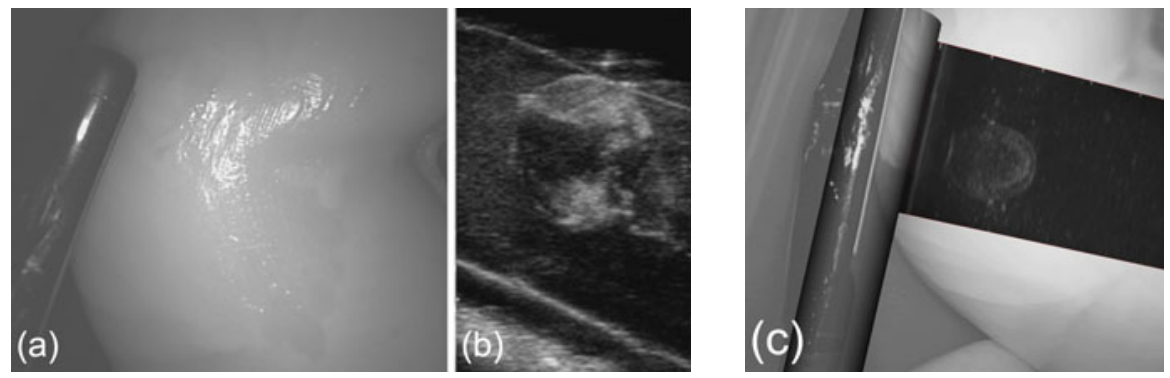

Fig. 1. (a)-(b) Separate video and US images, with the US probe visible in the video image. (c) Fused video and US images showing a tumour within a phantom.

\section{Materials and Methods}

\subsection{Fusion System}

Our image fusion platform used the Aurora magnetic tracking system (MTS) (Northern Digital Inc., Waterloo, ON) to determine, in real time, the location of images from a stereoscopic camera and an US probe. A 6 degree-of-freedom MTS sensor was affixed to each tool. The camera was calibrated using Zhang's method [7] to determine its intrinsic and extrinsic properties. We adapted this method to integrate the camera with the MTS by determining the transform between the camera's optical origin and its sensor. The US was calibrated [8] to 
determine the transform between the center of the probe element array and its sensor. The tracking of the tools combined with calibration was the key to the fusion of the images 9. Using the real-time positions and orientations of the images, they were rendered in a common coordinate system to create the fusion.

The visualization was run on an x86-based desktop personal computer with dual quad core Xeon processors at $2.66 \mathrm{GHz}$ and $4 \mathrm{~GB}$ RAM, running Windows XP with an NVIDIA Quadro FX 4600 graphics card. We used an Intuitive Surgical stereoscopic laparoscope from Olympus plugged into a Snell \& Wilcox vision cart with a Kudos Plus TBS100 synchronizer. An Aloka SD-1700 US system was used with a $7.5 \mathrm{MHz}$ laparoscopic probe.

Validation. The accuracy of tool calibration and image fusion was demonstrated with a target localization experiment using a tracked point source target. The target was placed at 20 different locations arranged in a grid pattern throughout tracking space. Each imaging modality was used separately to locate the target. The camera was used between 40 and $60 \mathrm{~mm}$ from the target, a range within which it would be used clinically. We compared the target location as determined by each modality to the true location given by tracking the target, and to the location determined by the other modality. To calculate the $3 \mathrm{D}$ location of the target according to US, its 2D US image coordinates were multiplied by the calibrated US tool transform [10. Triangulation [11] was used to compute the location of the target according to the stereoscopic camera images. This task demonstrated how well the camera and the US were calibrated relative to the tracking space coordinate system, as well as to each other.

\subsection{Phantom Study}

A study using kidney mimicking phantoms was performed to compare the fusion system mentioned in the previous section to the conventional visualization system that is currently implemented in the OR.

Phantom Development. Polyvinyl alcohol (PVA) was used in this study to create tissue mimicking phantoms. PVA, a synthetic polymer with high tensile strength and flexibility, is suitable for creating phantoms due to its nontoxic properties, ease of preparation and excellent echogenicity. The concentration of PVA relative to water and the number of freeze/thaw cycles (FTC) determines the rigidity and density of the final product. We used a $10 \%$ by mass solution of PVA and water. The PVA undergoes FTCs (12 hours freeze and 12 hours thaw per cycle) to create a cryogel (PVA-C). The process of creating PVA-C and the effect of the number of FTCs on its properties have been reported previously [12.

Our phantoms consisted of two components: tumour and normal parenchyma. Each was subject to a different number of FTCs so that they differed in density, enabling them to be distinguished under US. A custom designed mold was used to create $19.05 \mathrm{~mm}$ diameter spheres. Gadolinium contrast agent was added to the PVA of the tumours for enhanced magnetic resonance imaging (MRI) visualization. The tumour-replicating spheres were frozen and thawed twice, then removed and placed into a larger kidney-sized mold. After the tumour phantoms 
were inserted, the larger mold was filled with PVA, and the entire system was subject to one FTC. In total, the tumour-mimicking PVA underwent three FTCs while the normal renal parenchyma-mimicking PVA underwent one cycle. The difference in density between tumour and normal tissue resulted in realistic contrast under US (Fig. 1 b). In addition to realistic imaging qualities, the phantoms were realistic in texture, as determined by our clinical collaborators.
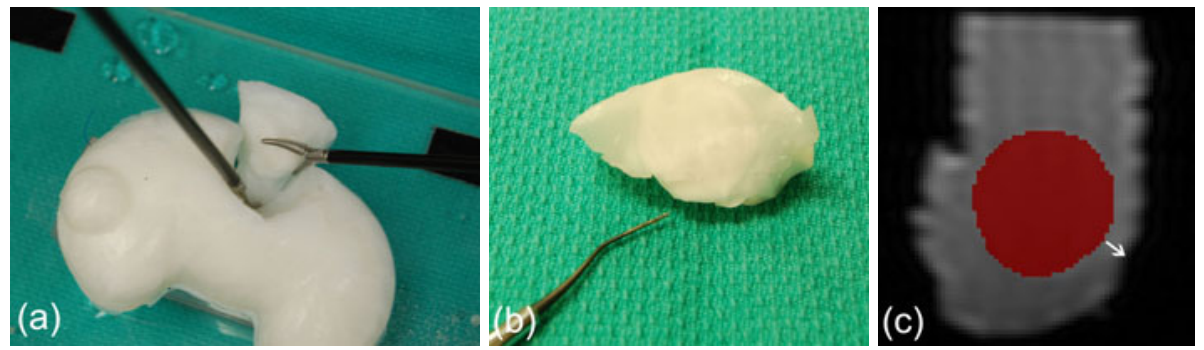

Fig. 2. (a) Tumour excision from phantom. (b) Excised segment. (c) MRI scan of excised section with segmented tumour (in red); white arrow denotes minimum margin.

User Study Procedure. We designed a task using the PVA-C phantoms to determine how well a surgeon is able to resect tumours under the guidance of fused video and US. The task replicated renal tumour resection using minimally invasive surgery (MIS) tools. This task was performed under the guidance of the conventional OR system with video and US displayed separately (conventional), the fused images presented in 2D (2D fusion), and the fused images presented in $3 \mathrm{D}$ (3D fusion). All of the visualizations were viewed on a novel autostereoscopic monitor (Dimension Technologies Inc., Rochester, NY) that can switch between 2D and 3D mode, eliminating any effect that may arise from variations in resolution and brightness caused by using a different monitor for each mode.

The resections were performed by a single experienced surgeon, who was instructed to resect the tumours as he would in the OR (Fig. 2a-b) and to aim for a $5 \mathrm{~mm}$ margin. For this experiment, we focused on endophytic tumours (completely beneath the organ surface) because they are the most difficult to operate on. Multiple resections were performed, each time under the guidance of one of the three visualization systems. The order in which the visualization systems were presented was randomized to minimize learning effects. A laparoscopy box trainer (Fig. 3a-b) was used to replicate the minimally invasive nature of the procedure. The phantom was placed inside the box trainer and laparoscopic tools were inserted through ports on the surface. The tools provided to the surgeon included laparoscopic scissors, graspers, marker (used in place of cautery for marking the phantom surface during planning), US probe, and camera, arranged in a similar manner to the setup in the OR (Fig. 3r). The US monitor was occluded when the fusion environment was being used.

Performance Measures. Performance was assessed by measuring the procedure time and the accuracy of margin achievement. A single factor analysis of 

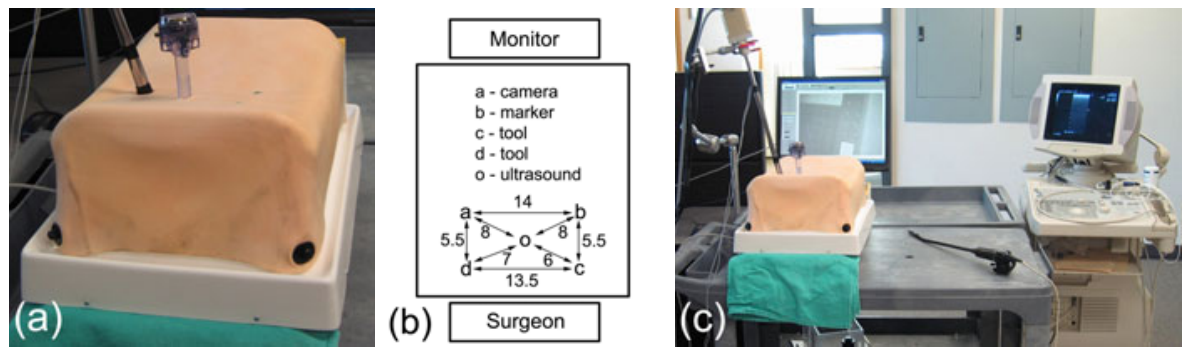

Fig. 3. (a) Laparoscopy box trainer used to replicate MIS environment. (b) Experiment port placement, numbers indicate distance in centimetres. (c) Experiment setup.

variance (ANOVA) was performed (GraphPad Prism 5.0) to determine significant differences in procedure duration between guidance modalities.

Time: The total procedure time was broken down into the planning stage and the excision stage. The planning stage included using the US to localize the tumour and using a marker to mark the surface of the phantom where the incision was to be made. The excision stage constituted the remainder of the procedure.

Accuracy: To evaluate margins, the excised sections were scanned with MRI. A T1 weighted sequence (T1 FLAIR) on a 3T GE MR750 Discovery scanner was used with the following parameters: TR $=2300 \mathrm{~ms} ; \mathrm{TE}=6.528 \mathrm{~ms} ; \mathrm{TI}=850 \mathrm{~ms}$; frequency $\mathrm{FOV}=12.8 ; \mathrm{NEX}=1$; echo train length $=2 ; \mathrm{BW}=31.25$; slice thickness $=1.0 \mathrm{~mm}$; and spacing $=0.2 \mathrm{~mm}$. The gadolinium contrast agent in the tumour PVA made it distinguishable from the normal parenchyma. This improved contrast allowed us to segment the tumours for post-operative evaluation. The volume of each segmented tumour was determined to validate the segmentation.

Clinically, margins are reported as positive (tumour extending to the edge) or negative (no cancer cells between the tumour and the edge). Some pathologists report the minimum margin from cut edges, although this is not consistent between all cases. We examined the MRI volumes of the excised sections and measured the shortest distance between the tumour and a cut edge to replicate the clinical scenario (Fig. 2k). We also reported whether the tumour boundary was exposed, indicating the potential for a positive margin.

\section{Results}

\subsection{Fusion System Validation}

The target localization error (Mean \pm Standard Error of the Mean(SEM)) was $1.20 \pm 0.08 \mathrm{~mm}$ for the camera, $1.85 \pm 0.14 \mathrm{~mm}$ for the US, and $2.38 \pm 0.11 \mathrm{~mm}$ between the camera and the US. Furthermore, a positive correlation $(r=0.86)$ was found between the error and the distance from the camera to the target, with the error being minimized in the camera focal range. 
Table 1. Resection times (Mean \pm SEM), in seconds

\begin{tabular}{|c|c|c|c|}
\hline & Planning & Excision & Total \\
\hline Conventional & $113.5 \pm 21.2$ & $331.6 \pm 49.8$ & $445.1 \pm 68.0$ \\
2D Fusion & $80.62 \pm 6.1$ & $393.8 \pm 62.9$ & $474.4 \pm 64.5$ \\
3D Fusion & $104.9 \pm 11.7$ & $541.9 \pm 86.5$ & $646.0 \pm 88.1$ \\
\hline
\end{tabular}

Table 2. Minimum margin thickness, in millimetres

\begin{tabular}{|c|c|c|c|}
\hline & Mean & SEM & Max \\
\hline Conventional & 2.10 & 0.89 & 6.05 \\
2D Fusion & 1.11 & 0.39 & 2.22 \\
3D Fusion & 1.76 & 0.79 & 5.10 \\
\hline
\end{tabular}

\subsection{Phantom Study}

The surgeon performed the task 6 times under each guidance modality, for a total of 18 trials. We report procedure times in Table 1 and margin accuracy in Table 2. These results show an improvement in the planning stage using 2D and $3 \mathrm{D}$ fusion. The tumour boundary was exposed in one case with the conventional system, in two cases with $2 \mathrm{D}$ fusion and in two cases with $3 \mathrm{D}$ fusion.

\section{Discussion}

The accuracy of the fusion is clinically sufficient as it allows surgeons to achieve acceptable margins without violating the tumour boundary. Furthermore, the accuracy of the MTS is on the order of $1-2 \mathrm{~mm}$ within our working volume, therefore our accuracy is also acceptable from an engineering angle.

The user experienced a $29 \%$ decrease in planning time with $2 \mathrm{D}$ fusion and an $8 \%$ decrease with $3 \mathrm{D}$ fusion over the conventional system $(\mathrm{p}=0.28)$. Excision times using the fusion systems were greater than with the conventional system, with the user being $16 \%$ slower with $2 \mathrm{D}$ fusion and $39 \%$ slower with $3 \mathrm{D}$ fusion $(\mathrm{p}=0.11)$. Overall, this led to a total time that was $6 \%$ slower with $2 \mathrm{D}$ fusion and $31 \%$ slower with $3 \mathrm{D}$ fusion compared to the conventional system $(p=0.15)$. Across all visualizations and procedure stages, time decreased with an increasing number of trials. This suggested a learning effect, which we expect will translate to improved procedure times with additional training.

The longer excision times with the fusion systems were due to latency in the visualization, especially with the second video image added for $3 \mathrm{D}$ fusion. The video was synchronized with the MTS, therefore its refresh rate was limited by tracking frequency. Our implementation would benefit from upgrades in the tracking system to provide a sufficiently high sampling rate when a greater number of tools is plugged in. With adequate hardware and software optimization, the excision times should be consistent over all visualizations, and the decreased planning time using the fusion systems should result in a decrease in overall procedure time. With such optimization, we suspect an even greater improvement 
in the planning time when using fusion. Clinically, the tumour resection is under 10 minutes. In all cases except for one conventional, one $2 \mathrm{D}$ fusion and two 3D fusion examples, our total time was less than 10 minutes.

Psychophysical considerations must still be addressed when overlaying images from multiple modalities, particularly when using stereoscopic images, a factor that contributed to the longer times with $3 \mathrm{D}$ fusion. Further work is required in optimizing the display of the images to make their combination convincing. Image processing [13] needs to be performed to remove ambiguity as to where the US beam lies with respect to the organ surface shown in the video, and to provide a more realistic environment. In addition, the surgeon reported occasionally losing the $3 \mathrm{D}$ view when he moved, forcing him to become reacquainted with the stereoscopic monitor, therefore an improved display method is desired.

Margin thickness varies, both in real procedures and in our study, which makes systematic comparison between cases difficult. Results show that the surgeon maintained a relatively fixed accuracy and decreased procedure planning time. We found that tumour boundary exposure occurred with all visualization systems. However, positive margins are not necessarily an indication for disease recurrence, as many of the tumours removed with partial nephrectomy are well encapsulated (reflected in our phantom model) and it is safe to cut along the tumour edge to retain more tissue, as long as the tumour is not violated.

In addition to the reduction in procedure planning time that we achieved, we aim to reduce overall procedure time and provide a means for the surgeon to gain consistent margins of a specified thickness. These goals will be achieved via improvements in the visualization through system optimization and image enhancements. The necessary improvements were determined through identification of constraints, from both observation and surgeon feedback, encountered in clinical practice that can affect the procedure. Future studies will include evaluating the performance of surgeons with varying levels of training, and additional trials for stronger statistical power.

\section{Conclusions}

We evaluated the use of fused US and video images for tumour resection during minimally invasive partial nephrectomy. This preliminary study demonstrated system usability and found an improvement in resection planning times using fusion. Optimization of the system components motivated by limitations encountered during our experimental procedures, and enhancement of the images to address psychophysical considerations, is expected to inspire faster overall procedure times and a more effective method of establishing margins.

\section{Acknowledgements}

The authors thank Kevin Wang and Cyndi Harper-Little for help with MRI scanning, and Dr. Usaf Aladl, Cristian Linte and Dr. Elvis Chen for discussion. We acknowledge funding from Cancer Imaging Network of Ontario. 


\section{References}

1. Gill, I.S., Desai, M.M., Kaouk, J.H., Meraney, A.M., Murphy, D.P., Sung, G.T., Novick, A.C.: Laparoscopic partial nephrectomy for renal tumor: duplicating open surgical techniques. The Journal of Urology 167(2), 469-476 (2002)

2. Sutherland, S.E., Resnick, M.I., Maclennan, G.T., Goldman, H.B.: Does the size of the surgical margin in partial nephrectomy for renal cell cancer really matter? The Journal of Urology 167(1), 61-64 (2002)

3. Leven, J., Burschka, D., Kumar, R., Zhang, G., Blumenkranz, S., Dai, X., Awad, M., Hager, G.D., Marohn, M., Choti, M., Hasser, C., Taylor, R.H.: DaVinci canvas: a telerobotic surgical system with integrated, robot-assisted, laparoscopic ultrasound capability. In: Duncan, J.S., Gerig, G. (eds.) MICCAI 2005. LNCS, vol. 3749, pp. 811-818. Springer, Heidelberg (2005)

4. Feuerstein, M., Reichl, T., Vogel, J., Traub, J., Navab, N.: New approaches to online estimation of electromagnetic tracking errors for laparoscopic ultrasonography. Computer Aided Surgery 13(5), 311-323 (2008)

5. Boritz, J., Booth, K.S.: A study of interactive 3D point location in a computer simulated virtual environment. In: Proceedings of the ACM symposium on Virtual reality software and technology, Lausanne, Switzerland, pp. 181-187. ACM, New York (1997)

6. Linte, C.A., Wiles, A., Moore, J., Wedlake, C., Peters, T.M.: Virtual realityenhanced ultrasound guidance for atrial ablation: in vitro epicardial study. In: Metaxas, D., Axel, L., Fichtinger, G., Székely, G. (eds.) MICCAI 2008, Part II. LNCS, vol. 5242, pp. 644-651. Springer, Heidelberg (2008)

7. Zhang, Z.: Flexible camera calibration by viewing a plane from unknown orientations. In: Proceedings of the 7th International Conference on Computer Vision, Kerkyra, Greece, pp. 666-673. IEEE Computer Society Press, Los Alamitos (1999)

8. Muratore, D.M., Galloway, R.L.J.: Beam calibration without a phantom for creating a 3D freehand ultrasound system. Ultrasound in Medicine \& Biology 27(11), 1557-1566 (2001)

9. Cheung, C.L., Wedlake, C., Moore, J.T., Pautler, S.E., Ahmad, A., Peters, T.M.: Fusion of stereoscopic video and laparoscopic ultrasound for minimally invasive partial nephrectomy. In: Miga, M., Wong, K. (eds.) Medical Imaging 2009: Visualization, Image-Guided Procedures and Modeling, Lake Buena Vista, FL, USA. SPIE 2009, vol. 7261, pp. 7261-7209 (2009)

10. Wiles, A.D., Moore, J., Linte, C.A., Wedlake, C., Ahmad, A., Peters, T.M.: Object identification accuracy under ultrasound enhanced virtual reality for minimally invasive cardiac surgery. In: Miga, M., Cleary, K.R. (eds.) Medical Imaging: Visualization, Image-Guided Procedures, and Modelling, San Diego, CA, USA. SPIE 2008, vol. 6918, p. 39180E (2008)

11. Hartley, R., Zisserman, A.: Multiple view geometry in computer vision, 2nd edn. Cambridge University Press, Cambridge

12. Surry, K.J.M., Austin, H.J.B., Fenster, A., Peters, T.M.: Poly(vinyl alcohol) cryogel phantoms for use in ultrasound and MR imaging. Physics in Medicine and Biology 49(24), 5529-5546 (2004)

13. Lerotic, M., Chung, A.J., Mylonas, G., Yang, G.: pq-space based non-photorealistic rendering for augmented reality. In: Ayache, N., Ourselin, S., Maeder, A. (eds.) MICCAI 2007, Part II. LNCS, vol. 4792, pp. 102-109. Springer, Heidelberg (2007) 